\title{
Slow journalism en Colombia: un estudio sobre las demandas de su audiencia y sus retos
}

\section{Guillermo Gurrutxaga Rekondo ${ }^{1}$ Amaia Álvarez Berastegi² Antxoka Agirre Maiora ${ }^{3}$}

Recibido: 26/01/2020

Aprobado por pares: 24/02/2020
Enviado a pares: $27 / 01 / 2020$

Aceptado: 08/03/2020

DOI: $10.5294 /$ pacla.2021.24.2.3

Para citar este artículo / to reference this article / para citar este artigo Gurrutxaga, G., Álvarez y A. Agirre, A. (2021). Slow journalism en Colombia: un estudio sobre las demandas de su audiencia y sus retos. Palabra Clave, 24(2), e2423. https://doi. org/10.5294/pacla.2021.24.2.3

\section{Resumen}

Este artículo presenta algunas claves de la situación actual del periodismo reposado digital o digital slow journalism (SJ) en el contexto colombiano. Para ello, analiza los resultados de una investigación sobre audiencias y estudia la óptica de medios encuadrados en esta corriente periodística. El artículo pone en común, por un lado, los resultados de una encuesta dirigida a la población colombiana realizada en julio de 2019 y cuyo objeto fue conocer las opiniones y hábitos de consumo del periodismo reposado. Destaca, entre sus resultados, el elevado conocimiento que existe en el país con respecto a la prensa narrativa. De hecho, el $56 \%$ de los lectores de prensa digital colombianos ha accedido alguna vez a ella. Por otro lado, al mismo tiempo, el estudio contrasta los intereses, percepciones y demandas del público con la respuesta dada por dos medios de SJ colombianos, Arcadia y La Silla Vacía, a través de un estudio cualitativo y comparado. La principal

\footnotetext{
$\triangle$ https://orcid.org/0000-0002-6770-979X. Universidad del País Vasco, España. guillermo.gurrutxaga@ehu.eus https://orcid.org/0000-0002-5826-6552. Universidad Pública de Navarra, España. amaia.alvarez@unavarra.es

https://orcid.org/0000-0003-0470-936X. Universidad del País Vasco, España. antxoka.agirre@ehu.eus
} 
conclusión es que los lectores colombianos valoran este tipo de periodismo (le otorgan una calificación de 4 puntos sobre 5 ) y hay un $58 \%$ de lectores que se manifiesta dispuesto a pagar por su plus de calidad. A pesar de los problemas de financiación a los que se enfrentan estos medios, la investigación vislumbra un nicho de mercado creciente para el SJ en Colombia.

\section{Palabras clave (Fuente: tesauro de la Unesco)}

Periodismo latinoamericano; periodismo narrativo; periodismo reposado; slow journalism; La Silla Vacía; Arcadia; prensa; prensa de información diaria; publicación seriada. 


\section{Slow Journalism in Colombia: A Study on the Demands of Its audience and Its Challenges}

\section{Abstract}

This article analyzes some keys to the current situation of digital slow journalism (SJ) in the Colombian context. It discusses the results of an audience study and explores the viewpoint of the media belonging to this journalistic trend. Besides, it shares a survey of the Colombian population conducted in July 2019 whose purpose was to know SJ opinions and consumption habits. The country's high knowledge of the narrative press stands out from the results. Actually, $56 \%$ of Colombian digital press readers have accessed it at some point. This study also contrasts the public's interests, perceptions, and demands with the response given by two Colombian SJ media, Arcadia and La Silla Vacía, through a comparative qualitative method. The chief conclusion is that Colombian readers value this type of journalism (rating it 4 out of 5), and $58 \%$ of readers say they are willing to pay for its extra quality. Despite the financing problems these media face, the research envisages a growing market niche for $\mathrm{SJ}$ in Colombia.

\section{Keywords (Source: Unesco Thesaurus)}

Latinamerican journalism; narrative journalism; slow journalism; La Silla Vacía; Arcadia; press; newspaper press; periodicals. 


\section{Slow journalism na Colômbia: estudo sobre as demandas de sua audiência e seus desafios}

\section{Resumo}

Neste artigo, são analisadas algumas particularidades da situação atual do jornalismo lento (digital slow journalism - SJ) no contexto colombiano. Para isso, analisam-se os resultados de uma pesquisa sobre audiências e estuda-se a ótica de meios delimitados nessa corrente jornalística. Neste artigo, coloca-se em comum os resultados de uma enquete dirigida à população colombiana realizada em julho de 2019 e cujo objetivo foi conhecer as opiniões e os hábitos de consumo do jornalismo lento. Destaca-se, entre seus resultados, o elevado conhecimento que há no país a respeito da imprensa narrativa. De fato, $56 \%$ dos leitores de imprensa digital colombianos já acessaram alguma vez a ela. Ao mesmo tempo, no estudo, contrastam-se interesses, percepções e demandas do público com a resposta dada por dois meios de SJ colombianos, Arcadia e La Silla Vacía, por meio de um estudo qualitativo e comparado. A principal conclusão está em que os leitores colombianos valorizam esse tipo de jornalismo (dão-lhe uma qualificação de 4 pontos sobre 5 ) e há $58 \%$ de leitores que se manifestam dispostos a pagar por seu plus de qualidade. Apesar dos problemas de financiamento que esses meios enfrentam, a pesquisa vislumbra um nicho de mercado crescente para o SJ na Colômbia.

\section{Palavras-chave (Fonte: tesauro da Unesco)}

Jornalismo latino-americano; jornalismo narrativo; jornalismo lento; slow journalism; La Silla Vacía; Arcadia; imprensa; imprensa de informações diárias; publicação seriada. 
El periodismo narrativo se abre paso como alternativa a la instantaneidad que predomina en internet. El contexto digital ha llegado a realizar una simbiosis entre el periodismo y la última hora, lo que ha ido relegando el viejo oficio de contar historias a una desaforada búsqueda del titular. Distintas investigaciones (González-Gorosarri, 2011; Ramírez et al., 2014; Gómez, 2009; 2015) advierten del deterioro de la calidad informativa. Aspectos como la competencia con las redes sociales han conllevado que muchos medios se hayan convertido en correa de transmisión de mentiras en una etapa marcada por la posverdad (Waisbord, 2018). Aunque la tecnología acarrea nuevos desafíos, la preocupación y el descontento por la falta de credibilidad de los medios no es nueva. De ahí que la reacción a los peligros de esa inmediatez reivindique la recuperación de los viejos principios periodísticos, una corriente que hoy llamamos slow journalism (SJ).

El periodismo narrativo, reposado o slow journalism constituye una tendencia que hace frente al ritmo acelerado impuesto por el mundo digital actual. Esta corriente tiene un largo recorrido tanto en América Latina como en el específico caso de Colombia, donde el realismo mágico de García Márquez ha influido en la elaboración de una tradición periodística que, en ocasiones, se ha acercado al periodismo literario y que hoy en día reivindica esa naturaleza narrativa también en el mundo digital (Sierra y López, 2016). La nueva crónica latinoamericana sorprende al lector con nuevas hibridaciones de géneros y con un nuevo periodismo narrativo (Sierra y López, 2016, p. 922) que ayuda a explicar problemas complejos (Palau, 2018).

Autores como Rodríguez y Albalad (2012) y Sierra (2012) hablan abiertamente de la existencia de un nuevo boom del periodismo narrativo latinoamericano, sugiriendo un cierto paralelismo con el boom de los años 50 y 60 del siglo XX, que precedieron al nuevo periodismo norteamericano (Wolfe, 1973). Otros autores, como Lago y Callegaro (2012) y Bonano (2014), subrayan que las "nuevas tendencias" son un fenómeno en alza en el continente americano. Colombia, fiel a su histórica tradición literaria y periodística (Hoyos, 2009; Jaramillo, 2012; Lago y Callegaro, 2012), no se ha quedado atrás en este auge del periodismo slow o narrativo y, tal y como 
señala Puerta, el país ha continuado retratando la sociedad a través de la crónica, "uno de los géneros que más ha aportado al desarrollo de la narrativa en Colombia y América Latina” (2011, p. 47). Este artículo pone de relieve cómo en los últimos años el país cafetero ha acogido la creación de nuevos proyectos periodísticos que forman parte de este periodismo narrativo latinoamericano y que, en el ámbito global, ubicamos dentro de lo que se ha denominado slow journalism.

A pesar de que el término slow journalism fue acuñado por primera vez en 2007 por la autora Susan Greenberg, no existe unanimidad en torno a él. Desde la academia se utilizó en un principio el término nuevo periodismo y otros como periodismo informativo de creación (Bernal y Chillón, 1985), y en la actualidad hay autores que utilizan indistintamente periodismo lento, periodismo narrativo y periodismo literario (Rodríguez y Albalad 2012). Para Le Masurier (2015), el periodismo slow es simplemente un tipo de periodismo "bueno, limpio (ético, transparente) y justo". En la industria profesional el término comenzó a cuajar hacia 2009, cuando publicaciones de la talla de Philadelphia Inquirer, Boston Globe, Huffington Post, Times y Forbes comenzaron a referirse al término slow media (Rauch, 2011). En el ámbito de habla castellana, la filosofía del slow journalism conecta con el periodismo literario, que, según indica López Pan (2010, p. 101), ha abordado los géneros de la crónica, la entrevista, el artículo de opinión, la naturaleza narrativa, anecdótica o ensayística, y la columna, además del reportaje novelado. No existe, por lo tanto, un modelo único para elaborar periodismo narrativo o reposado (Le Masurier, 2016; Barranquero y Jaurrieta, 2016) y en la actualidad, de hecho, cada vez es más común ver el periodismo narrativo en formato de libro y apartado de los medios convencionales (Cuartero, 2019).

Numerosos autores exploraron este concepto durante la primera fase de consolidación del periodismo digital entre 2005 y 2010 (De Pablos y Mateos, 2004; Pellegrini y Múgica, 2006; Amado, 2007; Rosenberg, 2008; Herrscher, 2009; Gripsrud y Weibull 2010). Más recientemente, la investigación académica sobre el concepto de periodismo lento ha revivido como 
respuesta a la inmediatez que predomina en el sector (Gómez, 2015). Así, los estudios sobre periodismo narrativo elaborados desde 2010 demuestran, entre otras cuestiones, que esta corriente periodística puede adaptarse a los diversos soportes del mundo digital (Rodríguez y Albalad, 2012; Greenberg, 2012), el fotoperiodismo (Mendelson y Creech, 2016) y el sector audiovisual más amplio (Albalad y Rodríguez, 2015; Bishop, 2015; Ball, 2016). En los últimos años, de hecho, hablamos del arranque de una nueva fase periodística en la que vuelven a primar los principios del slow journalism (Barranquero, 2013; Angulo, 2014; Llop, 2014; Benaissa, 2017; Goikoetxea y Ramírez, 2019). Por lo tanto, si bien la tradición slow no constituye una novedad, el auge de la inmediatez y la pérdida de la calidad periodística han propiciado que esta tendencia resulte necesaria para incrementar la credibilidad del oficio.

Existe todavía una necesidad de teorización del slow journalism en la era digital más reciente. Neveu (2016), por ejemplo, propone clasificar este tipo de periodismo en tres subgrupos: exploratorio, narrativo y movilizador. También con el objeto de profundizar en este fenómeno, Rosique y Barranquero explican que "el periodismo slow emerge como reacción a la novedad, brevedad e instantaneidad, e invita a repensar los tiempos necesarios para producir y consumir una información rigurosa, creativa y de calidad” (2015, p. 453). Una de las características del periodismo slow o lento es que tiene el objeto de atraer a una audiencia especializada (Drok y Hermans, 2016) a un nicho de mercado, y este es precisamente también el objetivo fundacional tanto de Arcadia como de La Silla Vacía, los dos medios analizados en este estudio, ya que se formaron como medios de información cultural y de actualidad política, respectivamente.

Otra de las características que comparten los medios de esta corriente slow es la falta de un modelo de negocio claro que sea viable y sostenible. Así, este tipo de publicaciones aún carecen de "estrategias de marketing para asegurar su futuro a mediano y largo plazo, y [las digitales] no terminan de aprovechar los recursos de la web para potenciar los contenidos y transformar las versiones electrónicas en plataformas de mayor relevancia 
informativa” (Rodríguez y Albalad, 2012, p. 289). De ahí que este artículo analice también las estrategias de Arcadia y La Silla Vacía para hacer frente al reto de la financiación. Siguiendo la tendencia que apunta Dowling (2016), estos dos medios colombianos han intentado huir de la dependencia de la publicidad tradicional y han implementado nuevas estrategias para promover los contenidos patrocinados y estrechar la relación con sus autores y audiencias.

\section{Metodología}

Esta investigación forma parte de un proyecto más amplio financiado por el Ministerio de Economía y Empresa (Mineco) del gobierno de España ${ }^{4}$ que analiza el periodismo reposado digital en español. En una primera fase, se realizó una revisión bibliográfica de referencias y estudios de caso de este tipo de medios digitales relacionados con los descriptores "slow journalism", "periodismo reposado", "periodismo narrativo", "periodismo narrativo digital" y "slow media”. Utilizando la plataforma de Google y la base de datos de Scopus (SJR), se hizo una primera selección inicial de 30 portales de periodismo narrativo que a la vez fueron analizados siguiendo los siguientes criterios: presencia de textos narrativos, desarrollo del contexto de los hechos y de los protagonistas en los textos publicados, pluralidad de los géneros utilizados (crónicas, entrevistas, reportajes, perfiles, crítica, ensayos, opinión... ), grado de actualización de los contenidos, nivel de referencialidad del sitio (audiencia certificada, seguidores en redes sociales, etc.) y, por último, calidad del diseño de la página web. A cada uno de estos seis apartados se le adjudicó una puntuación que oscilaba entre 0 (nula aportación) y 5 (máxima), por lo que la puntuación máxima que se podía obtener era de 30 puntos. Por último, el equipo de investigación valoró como muy importante que la muestra reflejara un amplio equilibrio geográfico y temático. De este modo se obtuvieron los diez medios objeto de estudio: Jot Down, Letras Libres, 5W, Gatopardo, CTXT, Anfibia, La Silla Vacía, Yorokobu y Panenka.

4 Proyecto de investigación financiado por el Ministerio de Economía y Competitividad (Mineco). Ref.: CSO 201676020-R (AEI/Feder, UE). Entidad financiadora: Ministerio de Economía y Competitividad, Fondo Europeo de Desarrollo Regional (Feder), Proyectos I+D. Programa Estatal de Fomento de la Investigación Científica y Técnica de Excelencia. 


\section{Tabla 1. Webs de periodismo narrativo en español. Puntuación, país y ámbito temático}

\begin{tabular}{|c|c|c|c|}
\hline Revista & Puntuación & País & Ámbito temático predominante \\
\hline $\begin{array}{c}\text { Jot Down } \\
\text { www.jotdown.es }\end{array}$ & 26,54 & España & Cultura \\
\hline $\begin{array}{c}\text { Letras Libres } \\
\text { www.letraslibres.com }\end{array}$ & 25,5 & México & Cultura \\
\hline $\begin{array}{c}5 W \\
\text { www.revista5w.com }\end{array}$ & 24,5 & España & Pol. Internacional \\
\hline $\begin{array}{c}\text { Gato Pardo } \\
\text { www.gatopardo.com }\end{array}$ & 23,2 & México & Cultura \\
\hline $\begin{array}{c}\text { CtXt, Contexto y Acción } \\
\text { http://ctxt.es }\end{array}$ & 22,4 & España & Generalista (política) \\
\hline $\begin{array}{c}\text { Anfibia } \\
\text { www.revistaanfibia.com }\end{array}$ & 22,31 & Argentina & Generalista (política) \\
\hline $\begin{array}{c}\text { La Silla Vacía } \\
\text { www.lasillavacia.com }\end{array}$ & 22 & Colombia & P. Investigación \\
\hline $\begin{array}{c}\text { Arcadia } \\
\text { www.revistaarcadia.com }\end{array}$ & 21,88 & Colombia & Cultura \\
\hline $\begin{array}{c}\text { Yorokobu } \\
\text { www.yorokobu.es }\end{array}$ & 21,05 & España & Creatividad \\
\hline $\begin{array}{c}\text { Paneka } \\
\text { www.panenka.org }\end{array}$ & 20,88 & España & Cultura futbolística \\
\hline
\end{tabular}

Fuente: elaboración propia.

En una segunda fase se dio inicio a los estudios de caso de cada medio (Lindlof y Taylor, 2017). En este artículo ofrecemos los resultados de los dos medios colombianos seleccionados, Arcadia y La Silla Vacía, un análisis que hemos triangulado con los resultados de una encuesta realizada en ese mismo país sobre los hábitos de consumo de periodismo reposado. La elaboración de las encuestas, de hecho, constituyó la tercera fase de la obtención de datos para la investigación.

Robert K. Yin (1992) defiende el estudio de caso como una indagación empírica que utiliza múltiples fuentes de conocimiento para investigar un fenómeno actual dentro de un contexto de vida real. En el caso de Arcadia y La Silla Vacía, el estudio de caso implicó el análisis de los contenidos (web e impresos) de estos dos medios durante un periodo de seis meses (de julio a diciembre de 2018). Se llevó a cabo con estos criterios: 
grado de actualización de los contenidos, presencia de contexto en los textos publicados (sobre hechos y/o protagonistas), presencia de textos narrativos (historias que emocionen, iluminen...), pluralidad de los géneros utilizados (crónicas, entrevistas, reportajes, perfiles, crítica, ensayos, opinión...), nivel de referencialidad y/o influencia del sitio (audiencia certificada, seguidores en redes sociales...) y, por último, calidad del diseño de la página web. Tras el análisis de contenido de estos dos medios, se procedió a realizar entrevistas en profundidad en las redacciones que estos dos medios tienen en Bogotá con sus responsables periodísticos, económicos y tecnológicos. En el caso de Arcadia, las personas entrevistadas fueron su director Camilo Jiménez y su editora general Sara Malagón. En el de La Silla Vacía, su directora Juanita León y su coordinador comercial Pablo Isaza. Todas las entrevistas se llevaron a cabo entre julio y agosto de 2018. Los resultados de los estudios de caso se realizaron utilizando métodos comparativos (Palacios y Díaz, 2009) y técnicas cualitativas (Wimmer y Dominick, 1996, p. 146).

En una última fase se triangularon estos datos con los resultados de las encuestas. Fueron realizadas online por el Centro de Investigación y Estudios Sociales (CIES), una de las principales empresas demoscópicas en España. Las encuestas se realizaron en julio de 2019 en España, México, Argentina y Colombia, países en los que se sitúan las diez webs analizadas y que se han escogido como referenciales. En cada país la encuesta tuvo 500 participantes, ${ }^{5} \mathrm{y}$ a todos ellos se les pasó un cuestionario de 40 preguntas enfocado a conocer la opinión de la población respecto al periodismo reposado y a analizar los hábitos de consumo de medios que practican este tipo de periodismo. El cuestionario intercalaba tanto preguntas cuantitativas como cualitativas y se articulaba en torno a estos ejes temáticos: perfíl del lector: datos demográficos; hábitos de consumo de medios en general y de medios de slow journalism en particular; motivaciones/valores respecto al periodismo narrativo; preferencias en cuanto a contenidos y estilos narrativos; exigencia informativa, calidad y rigor;

5 El universo de las encuestas son habitantes de estos países con una edad comprendida entre los 18 y los 65 años y con acceso a internet. Se ha procurado que los porcentajes de hombres y mujeres sea parejo. Dentro de estos parámetros, el perfil de los participantes colombianos es el de un usuario mayoritariamente joven (48 \%, 18-34 años; 29\%, 50-65; y $23 \%, 35-50)$, con estudios universitarios (59\%) y que vive en una ciudad de más de 500.000 habitantes (57\%). 
participación/interactividad en medios slow; grado de satisfacción con los medios slow consumidos.

\section{Resultados}

Según el resultado de las encuestas realizadas, 1 de cada 5 colombianos que alguna vez ha leído prensa digital afirma haber oído hablar del concepto de periodismo narrativo y más de la mitad ha accedido alguna vez a algún medio encuadrado entre los que apuestan por el slow journalism. En concreto, el $56 \%$. Colombia es el país que lidera el consumo de prensa narrativa por parte de las personas que alguna vez han leído prensa digital entre los cuatro donde se llevó a cabo la encuesta, y le siguen, a distancia, México, con el 38 \%; Argentina, con el $25 \%$; y España, con el $24 \%$.

¿Qué es lo que acerca al público colombiano a este tipo de periodismo? El acceso a opiniones críticas y la capacidad de análisis desarrollada a través de estos medios es lo que mencionan más de la mitad de las personas consultadas. De cerca le siguen la actitud reflexiva, la información de contexto o la independencia editorial.

\section{Tabla 2. Elementos que ofrece el periodismo narrativo. Posible respuesta múltiple.}

\begin{tabular}{|l|c|}
\hline Opinión crítica & $66 \%$ \\
\hline Actitud reflexiva & $49 \%$ \\
\hline Capacidad de análisis & $53 \%$ \\
\hline Calidad/claridad informativa & $39 \%$ \\
\hline Creatividad & $34 \%$ \\
\hline Información de contexto & $41 \%$ \\
\hline Independencia editorial & $40 \%$ \\
\hline Narraciones dinámicas & $38 \%$ \\
\hline Transparencia & $27 \%$ \\
\hline Especialización & $19 \%$ \\
\hline
\end{tabular}

Fuente: elaboración propia. 
Hay una conexión entre la prensa narrativa y los géneros periodísticos que más les gustan a las personas que la leen, puesto que la crónica (34\%), el análisis (28\%), el reportaje (16\%), la opinión (14\%) y la entrevista (5\%) son sus preferidos. Coincide esta visión del público colombiano con la filosofía de La Silla Vacía y Arcadia. Ambas acuden a los grandes géneros del periodismo, como la crónica y el análisis, para trasladar su profunda mirada de la realidad, pero la innovación forma parte también de su identidad, de tal manera que acuden a nuevas formas de contar historias. Arcadia recoge una amplia variedad de géneros $y$, a veces, dependiendo del tema, estos también se hibridan. Trabajan, sobre todo, reportajes, entrevistas, perfiles, análisis literarios, críticas y reseñas. Incluyen crónicas cortas, pero lo más habitual son los temas largos o "de largo aliento", tal y como explica su directora general Sara Malagón. El análisis es el género periodístico que prima en La Silla Vacía, con frecuencia combinado con el reportaje. La entrevista es el género predominante en su sección La Silla Académica. La directora y fundadora de La Silla Vacía, Juanita León, da relevancia también a lo que denomina "perfiles en profundidad”. La apuesta por este género llevó a la edición del libro Súperpoderosos: los protagonistas de 2014. León aboga por nuevos formatos narrativos que superen el esquema tradicional, como el del detector de mentiras. Y es que otros contenidos, como el listado del "Quién es quién: las conexiones del poder" que aparece en su portada, tampoco pueden encuadrarse en los géneros tradicionales. Sin embargo, enlaza con la idea de su directora de informar más mediante los datos que con el texto. Secciones como "Quién es quién” forman parte de las innovaciones que La Silla Vacía ha ido incorporando desde su nacimiento. Como lo fue la incorporación de información regional, a la que puede accederse a través de las pestañas de lo que León denomina "las sillas regionales": La Cachaca, La Caribe, La Pacífico, La Paisa, La Santandereana y La Sur, que nacieron, dice, de "una decisión política, acorde con el momento histórico que Colombia vivió”. En ese contexto surgieron otras iniciativas, como La Silla Llena o La Silla Académica, vinculadas con la necesidad de nuevas fuentes de financiación que no afectaran ni la independencia de La Silla Vacía ni su esencia. Define La Silla Llena como una red especializada de conocimiento, cada una de cuyas redes es patrocinada por un sponsor: "Hay como $600 \mathrm{ex}$ pertos inscritos y el año pasado [2017] escribieron 250 de esos expertos". 
Son 15 las redes de conocimiento sobre las que esos expertos escriben en la Silla Llena. Hay redes temáticas, como las denominadas Étnica, Verde o Mujeres, donde las cuestiones que se abordan están relacionadas, respectivamente, con la diversidad etnográfica, la ecología o el feminismo. Otras, como Caribe, Sur o Paisa, se centran en cuestiones vinculadas con distintas regiones. Tras cada una de estas redes hay una fundación u organización que la patrocina. También empresas, pero Pablo Isaza, su coordinador comercial, pone énfasis en que La Silla Vacía cuida que haya una coherencia entre su naturaleza y su filosofía. En esa misma estrategia se asienta La Silla Académica. Isaza la define como un producto exclusivo "para universidades, para centros que generan conocimiento y que publican contenido académico". En ella las universidades suscritas suben las reseñas de los papers y libros de temáticas cercanas a las que aborda la La Silla Vacía, así como las convocatorias a los eventos que organizan. La Silla Académica tiene su propia editora, que escoge a lo largo del año diez papers o libros por cada una de las cinco universidades afiliadas para hacerles, en palabras de Juanita León, "una traducción periodística: una entrevista al autor o un vídeo o una historia que agregue valor a la coyuntura”.

En esa apuesta de La Silla Vacía y Arcadia por la profundidad, la utilización de géneros interpretativos como el análisis conecta también con las preferencias del público colombiano, puesto que el 58\% de los lectores de prensa narrativa consultados en la encuesta llevada a cabo en ese país para la realización de esta investigación mencionan el de Opinión como género más leído. Le siguen Cultura, señalado por el $57 \%$, y Política, con el $55 \%$, precisamente los dos ámbitos temáticos en los que están especializados Arcadia y La Silla Vacía.

Los contenidos de Arcadia abarcan diferentes aspectos de la cultura, a la que no priva de un enfoque sociopolítico. En esa visión más amplia del fenómeno cultural y sus conexiones con otras cuestiones que afectan a la sociedad destacan los contenidos relacionados con el conflicto (desplazamiento, etc.), la diversidad cultural (comunidades afrocolombianas) y la perspectiva feminista. Su mirada sensible intenta también combinar la denominada alta cultura con nuevas manifestaciones, impulsadas, por ejem- 
plo, con las nuevas tecnologías, o creadas y dirigidas al margen de las élites. Los artículos de Arcadia destacan por su calidad, profundidad y el mimo con el que se elaboran. La filosofía de los contenidos está, por lo tanto, ligada al periodismo reposado, narrativo o slow journalism. Cada mes, Arcadia piensa bien los contenidos y planifica los próximos números con una mirada a mediano o largo plazo que no necesariamente coincide con eventos culturales. Esta característica slow de Arcadia se entiende como un elemento estratégico en el que se intenta ahondar cada vez más. Sara Malagón, su editora general, define el periodismo reposado como un periodismo que "no responde a las coyunturas" y tiene como elementos centrales "la planificación, el tiempo de dedicado y la extensión larga”, además de "la profundidad" y "el diseño", que trabajan enfocándolo en una lectura más reposada. Si el carácter mensual de su edición en papel condiciona su talante reposado, en internet sus actualizaciones se sitúan en la media de tres diarias. La estructura de la revista también responde a la filosofía slow. En este sentido, destaca la apuesta por un gran tema, un especial, cada mes.

\section{Figura 1. Portadas de Arcadia}

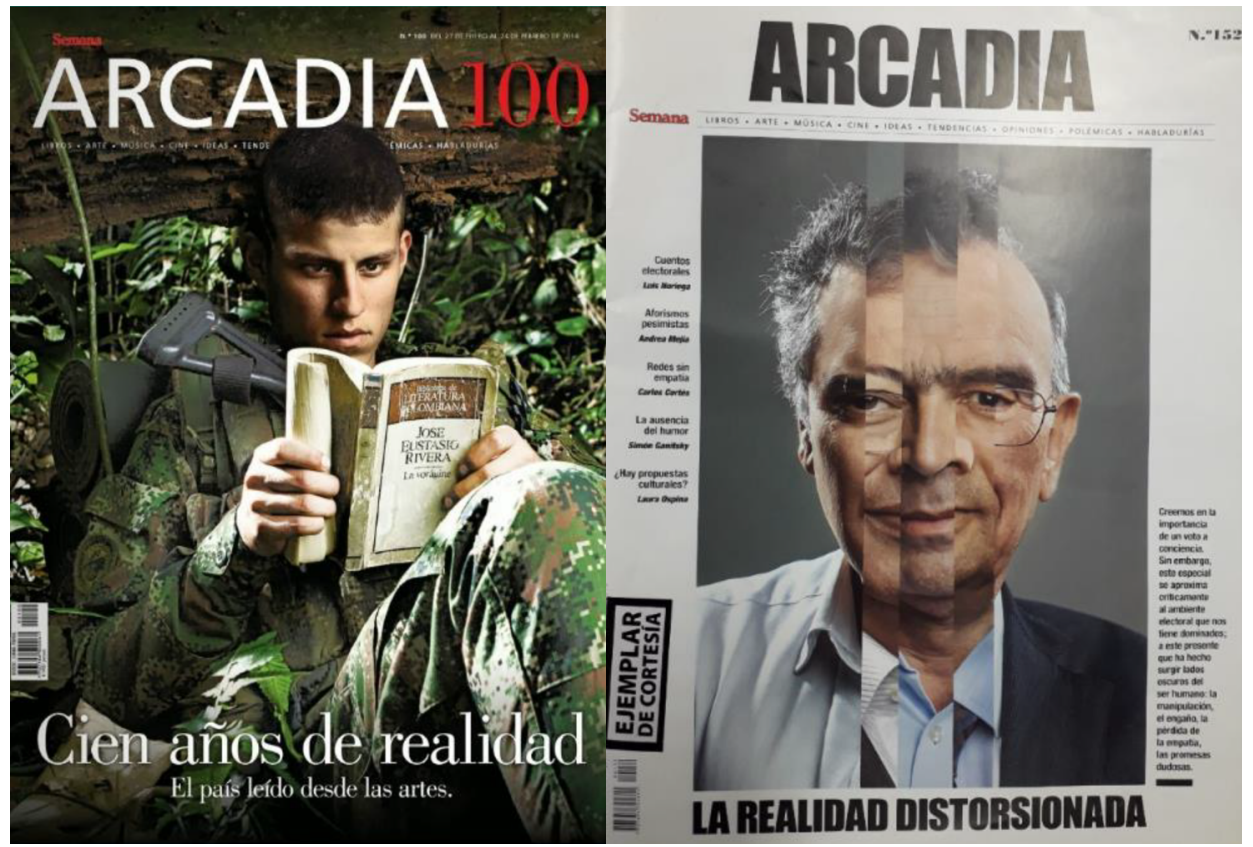

Fuente: Arcadia (núms. 100, febrero de 2014, y 152, junio de 2018). 
La elección del tema central es estratégica y la que más planificación conlleva en la confección de cada número. Las secciones varían en función del mes, habida cuenta de que cada número se entiende como un todo que requiere del diseño de su propia estructura. De todos modos, habitualmente mantiene el mismo esquema: Editorial, Portada (tema especial), Mutantes Digitales, Literatura, Música, Crónica, Críticas, Libros, Opinión. En otras ocasiones se añaden secciones como Cine y Filosofía. En el último año, además, se han incorporado otras secciones para dar un mayor espacio a la fotografía y a la cultura digital.

En La Silla Vacía la apuesta por el periodismo lento está relacionada, sobre todo, con la profundidad. León alude a un estudio efectuado en 2016 en el que se analizaron las 20 historias más leídas en La Silla Vacía y se concluyó que su lectura no dependía de la extensión del texto: "A nuestros usuarios no les importa si es larga o no" y sí, en cambio, "los personajes involucrados y el titular". De ahí su intento de "narrar gráficamente, quitar texto y narrar a partir de datos, de gráficos”. En ese sentido, León considera que la labor periodística está, más que "en las palabras lindas”, en las "llamadas", en las “comprobaciones", en los "documentos". En su opinión, la profundidad está muy relacionada con la verificación mediante fuentes diversas. Y en no hacer cálculos en cuanto al compromiso de que sus periodistas cuenten lo que sepan. La apuesta por la agenda propia intenta cubrir aspectos descuidados tanto por el poder como por la propia sociedad. Como paradigmático en este sentido, señala lo relacionado con las víctimas del conflicto armado, la igualdad de género o los temas medioambientales. Y para eso resulta esencial huir de la obsesión por la inmediatez. León cree haber encontrado el equilibrio en la inclusión habitual tres nuevos contenidos diarios, a los que se suma uno que se ofrece en cada Silla Regional. Las actualizaciones se efectúan en tres franjas horarias: el mediodía, la medianoche y la tarde, en torno a las 17.00 horas.

La Silla Vacía cumplió su primera década en 2019 manteniendo su clara apuesta por el periodismo digital. Arcadia, en cambio, nació en 2005 como suplemento cultural de la revista Semana. No dejó de distribuirse de forma separada e independiente hasta 2012. Aunque Malagón hace una 


\section{Figura 2. Captura de portada, 13 de octubre de 2018, menú Secciones, subsección Las Sillas}

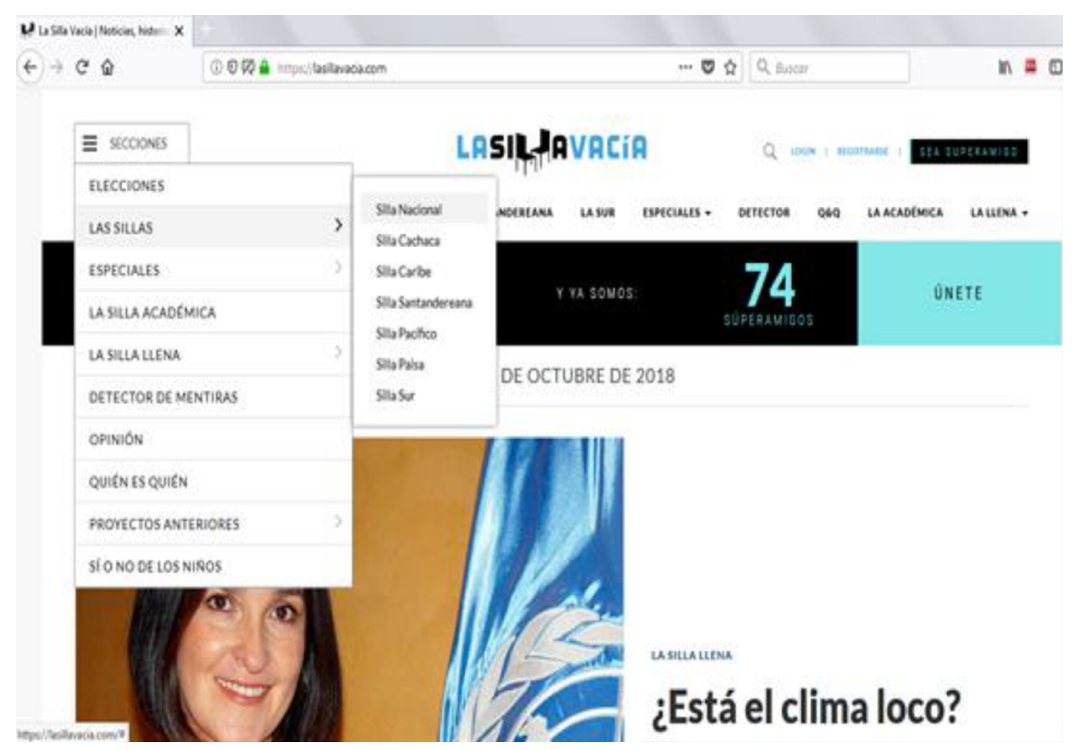

Fuente: La Silla Vacía.

identificación entre el papel y su carácter narrativo, el creciente peso de su web resulta un fiel exponente de su esfuerzo por adaptarse a los gustos y necesidad de un público, el colombiano, que, según la encuesta, se decanta claramente por la web a la hora de consumir prensa narrativa. El 69 \% de los usuarios lo utilizan, frente al $10 \%$ que acude al papel y el $21 \%$ que accede a ambos soportes. Otro de los datos que visibiliza la creciente fortaleza de las nuevas tecnologías está en que las redes sociales son el medio prioritario para informarse por parte de la audiencia colombiana. También son el medio más utilizado para recabar información, puesto que el $86 \%$ de los lectores de prensa digital acuden habitualmente a ellas. Arcadia contaba a finales de 2018 con 125.000 seguidores en Twitter. Los de Facebook se acercaban a los 170.000, cifras significativas en el nicho cultural. La revista también está en Instagram, Google Plus y Youtube. En octubre de 2018 La Silla Vacía se acercaba a 1.200.000 seguidores en Twitter(@lasillavacia) y los de Facebook eran 232.000. Tiene un canal en Youtube, con cerca de 20.000 personas suscritas. Estos datos son una muestra de la estrategia manejada por ambos proyectos para hacerse un hueco en las redes sociales 
y dar uso a la interactividad de estos formatos. En la búsqueda de la identidad propia, la edición digital de Arcadia intenta exprimir las herramientas que permiten la interacción con las audiencias. Además, el usuario de la web de Arcadia es más joven que el de la versión impresa, dado que la revista atrae todavía a un segmento de mediana edad y clase media-alta. En el caso de La Silla Vacía, la interacción con su público está configurada como "un diálogo", según la definición de Juanita León. Ella misma responde directamente, en su calidad de directora, a los comentarios formulados en torno a los artículos identificados como opinión que ella misma escribe en un espacio que define "como un blog".

Los datos de la encuesta validan esta estrategia, puesto que el $79 \%$ de los lectores de prensa digital colombiana muestran interés por los comentarios que acompañan a las noticias y el $35 \%$ interactúa con el medio o comparte su contenido con otros usuarios. En este sentido, hay otro dato que refuerza el papel que desempeña el propio público en la propia expansión del contenido de los medios narrativos: el $74 \%$ de los lectores de prensa narrativa digital acceden a él a través de las redes sociales, mientras que el $51 \%$ lo hace a través de la web del propio medio. Hay otro $28 \%$ de accesos a través de blogs. En cuanto al manejo de las cuentas de La Silla Vacía en Twitter y Facebook, León señala que "los periodistas hacen turnos" y cada dos horas participan de ese "diálogo" con el público. León considera que aún están "comenzando" en Instragram, donde en octubre de $2018 \mathrm{La}$ Silla Vacía acumulaba 33.000 seguidores. La actividad de este medio en las redes sociales también es estratégica. Y está, de igual manera, condicionada por la innovación. Las cifras recogidas por Google Analytics muestran un aumento imparable de su audiencia: la cifra de usuarios únicos alcanzada en 2017 (380.508) triplicó la alcanzada en 2010 (124.111), al año de su lanzamiento. En cuanto al tipo de dispositivos, las sesiones iniciadas desde un celular llegan a doblar a las de computador. En consonancia con estos datos de La Silla Vacía, la encuesta refleja que, entre la audiencia colombiana de prensa narrativa digital, el $86 \%$ utiliza el teléfono celular para acceder a los medios que consulta y el $58 \%$ lo hace con el portátil, mientras que el ordenador de mesa y la tableta o los lectores digitales se sitúan a gran distancia. El dato es coherente con ese perfil de público joven que León situaba en- 
tre los 25 y los 40 años. Su web indica que, "desafortunadamente", de cada tres personas que la leen solo una es mujer. Casi el $90 \%$ de sus visitantes lo hacen desde la propia Colombia. En cuanto a los vídeos, un análisis de los subidos a Youtube durante el mes de octubre de 2018 arroja una audiencia máxima de 15.000 visualizaciones y mínima de en torno a las 1.000. León no oculta la "obvia" importancia que otorga al tráfico. Sin embargo, matiza que no es el único indicador en el que reparan de cara al sostenimiento del proyecto: "Nos ponemos felices cuando alguien nos cita en una columna, cuando nos utilizan en un libro".

\section{Tabla 3. Dispositivos utilizados por las personas que leen prensa narrativa digital (posible respuesta múltiple)}

\begin{tabular}{|l|c|}
\hline Smartphone & $86 \%$ \\
\hline Portátil & $58 \%$ \\
\hline Ordenador de mesa & $37 \%$ \\
\hline Tablet & $21 \%$ \\
\hline E-readers & $25 \%$ \\
\hline
\end{tabular}

Fuente: elaboración propia.

Si las redes sociales son el medio más habitual y prioritario para informarse por los colombianos que leen prensa digital, son estos mismos medios digitales el segundo soporte en el listado. Entre los tradicionales, les sigue de cerca la televisión, mientras que la prensa impresa cierra la lista.

\section{Tabla 4. Medios habituales (posible respuesta múltiple) y medios prioritarios para informarse de la actualidad}

\begin{tabular}{|l|c|c|}
\hline & Medios habituales & Medio prioritario \\
\hline Prensa digital & $76 \%$ & $28 \%$ \\
\hline Prensa impresa & $31 \%$ & $2 \%$ \\
\hline Radio & $50 \%$ & $10 \%$ \\
\hline TV & $79 \%$ & $24 \%$ \\
\hline Redes sociales & $86 \%$ & $36 \%$ \\
\hline Otros & $1 \%$ & $1 \%$ \\
\hline
\end{tabular}

Fuente: elaboración propia. 
La preeminencia de lo digital se refleja también en que el $90 \%$ de los lectores que consumen o han consumido prensa narrativa considera que la inclusión de recursos audiovisuales y/o multimedia en los contenidos periodísticos facilita la comprensión de las noticias.

La respuesta a esa demanda del público se da, en el caso de Arcadia, mediante una cuidada calidad de sus recursos audiovisuales, equiparable a la de las fotografías de su edición impresa. Cuenta para elaborar su contenido con el equipo que lleva a cabo todos los vídeos que nutren las páginas web del Grupo Semana. Recurren con frecuencia a la elaboración de vídeos y a Facebook live para cubrir eventos culturales y difundir debates organizados por la propia revista. Asimismo, el audio y los podcasts forman parte de los ejes estratégicos de Arcadia, principalmente para atraer a los más jóvenes. La Silla Vacía también cuenta con vídeos de elaboración propia, caracterizados por su edición sencilla, en los que predomina el discurso frente a la imagen. Por último, hay también informaciones desarrolladas en podcast, en las que el texto escrito de la entrada es solo un elemento introductorio. En este sentido, León ve como contradictorio el escaso, aunque creciente, número de vídeos que aparecen en la Silla Vacía, algo que, en parte, atribuye a que la mayoría de las fuentes con que trata son anónimas, así como al costo que tienen.

El carácter slow de Arcadia y La Silla Vacía se vislumbra también en su estructura empresarial. El modelo de negocio periodístico, así como la industria en general, han cambiado de forma dramática en la última década (Gómez, 2009; Anderson, Bell y Shirky, 2013), y estos dos medios colombianos han sabido adaptarse. Tienen plantillas muy ajustadas. En el caso de Arcadia, tan solo tiene a cuatro personas en nómina: su director, la editora general, el responsable de la edición digital y la subdirectora comercial. El resto son colaboradoras o personas empleadas del Grupo Semana. La ausencia de este tipo de sinergias hace que la plantilla de La Silla Vacía aumente a un grupo de 18 personas, en su mayoría mujeres de entre 25 y 30 años, según el perfil aportado por Pablo Isaza, su coordinador comercial. Los dos proyectos desarrollan distintas iniciativas para cimentar un nuevo modelo económico menos dependiente de la publicidad tradicional. Com- 
parten su planificación estratégica a largo plazo, siempre coherente con los principios que subyacen a su línea editorial.

El carácter reposado de su información se corresponde, por lo tanto, con apuestas que huyen de estrategias cortoplacistas que buscan resultados inmediatos. En el caso de Arcadia, durante 2018 el ingreso por suscripciones llegó al $10 \%$ del total. Se plantean alcanzar el $80 \%$ en un plazo de entre ocho y diez años, "pero ese es un camino muy largo y muy difícil", apunta Camilo Jiménez, su actual director. En Arcadia la publicidad online solo supone, al día de hoy, el $5 \%$ del total de ingresos. La decisión clave para incrementar las suscripciones y reducir, por lo tanto, la dependencia respecto a la publicidad fue establecer el paywall y cerrar el acceso a la web, que anteriormente era totalmente gratuito. Junto con esta decisión (tomada en 2016 e implementada a finales de 2017) se dividieron los contenidos. Así, se distinguió entre los de pago, restringidos a los suscriptores, y los gratuitos. Ofrecen en la actualidad dos tipos de suscripciones mensuales: digital ( $\$ 55.000$ pesos colombianos o $€ 15,6$ euros $^{6}$ ) o digital e impresa $(\$ 69.000$ pesos colombianos o $€ 19,5$ euros).

Los contenidos gratuitos buscan atraer a nuevos lectores de pago. El cierre de contenidos en función del pago ha evolucionado y actualmente solo permite acceder a tres o cuatro contenidos completos de forma gratuita. Son específicamente creados para internet, puesto que del resto -los artículos volcados desde la edición impresa- solo se puede leer el primer párrafo de cada pieza. El sistema de paywall aún está en fase de prueba y sujeto a modificaciones. Desde su implementación el número de suscriptores "se ha visto incrementado". Según explica Jiménez, Arcadia ha sido "deficitaria algunos años desde su creación, muy frágil en sus primeros siete años de vida, y la crisis de la publicidad se dejó notar con especial ímpetu", pero el apoyo del Grupo Semana y el hecho de haber reinventando el proyecto están dado sus frutos. Arcadia busca nuevas fuentes de financiación coherentes con su identidad. En 2017 generó "más ingresos que nunca, pero también más gastos que nunca", apunta Jiménez. Poco a poco, se van acercando al equilibrio ideal. Los cambios y el proyecto son una apuesta

6 Teniendo en cuenta el precio de cambio de peso colombiano a euro, para el 14 de mayo de 2019. 
a largo plazo, "de fondo". Acude, entre otras fórmulas, a los denominados sponsored content. Así, instituciones públicas como el Ministerio del Interior, la Alcaldía de Cali, u organismos internacionales como la Organización Internacional de las Migraciones (OIM), bancos e incluso embajadas apoyan reportajes e iniciativas de Arcadia que ponen el foco en las víctimas del conflicto armado. Por otro lado, acude también al branded content, contenido pagado por una marca. Se autoimpone que aparezca completamente diferenciado (con otro color de fondo, etc.) para no confundir al lector. Ahora mismo, según apunta Jiménez, este tipo de publicidad es la que más está creciendo.

En el caso de la La Silla Vacía, su principal fuente de financiación proviene de la cooperación internacional, lo que incluye aportes de gobiernos como el de Gran Bretaña y fundaciones como Open Society y Ford. Por el contrario, la publicidad suponía en 2018 tan solo un $7 \%$ de los ingresos, uno de sus principales elementos diferenciadores con respecto a los medios tradicionales. Porque no está dispuesta a aceptar cualquier anuncio, sino que “debe aportar valor agregado", según matiza Pablo Isaza. Pone como ejemplo una campaña efectuada por Movistar Colombia, quien acudió a La Silla Vacía "para difundir su compromiso con la equidad de género": "Destacamos a las 100 mujeres que han transformado el país. E hicimos una investigación, con reportería, para resaltar a esas mujeres y armar el su perfil”. La página web de La Silla Vacía atribuye a este tipo de eventos el $13 \%$ de su financiación. El ya aludido $7 \%$ alcanzado mediante la publicidad es el mismo porcentaje que aportaron los Superamigos, un grupo de personas a los que La Silla Vacía ofrece algunas ventajas, pero que básicamente hacen entregas altruistas de dinero. En 2017 fueron 920. Cada uno de ellos hizo un aporte mínimo de $\$ 50.000$ pesos (aproximadamente $€ 15$ euros) y un máximo de $\$ 1.500 .000$ (unos $€ 450$ ). Tienen acceso a idéntico contenido que quienes no aportan nada, puesto que la totalidad de lo publicado por La Silla Vacía está disponible de forma gratuita en la web, sin necesidad de registro previo. En 2017 recaudó a través de sus Superamigos \$130.000.000 (€37.000), según los datos aportados por Pablo Isaza, quien sitúa la media entregada por cada donante en una cuantía cercana a los $\$ 100.000$ ( $€ 28$ euros). Por último, las cinco universidades que por su parte participan en La Silla Aca- 
démica contribuyeron al 6,5\% de los ingresos en 2018, segundo año consecutivo en el que La Silla Vacía consiguió beneficios.

Y es que, aunque no sin dificultades, el periodismo reposado va abriendo y consolidando su propio espacio entre el público colombiano. Asílo refleja la encuesta llevada a cabo en Colombia para esta investigación. De 0 a 5 , los colombianos que leen prensa narrativa le otorgan un 4 a su futuro, al igual que a su calidad. Y aunque solo el $14 \%$ de quienes alguna vez han accedido a prensa digital en Colombia están suscritos a un medio que apuesta por el slow journalism, el $49 \%$ muestra su disposición a pagar por el plus de calidad que este supone. No en vano, el $57 \%$ apuesta por que el periodismo se vuelque al slow journalism y lo haga a través de internet. Otra vez, la cifra más alta de entre los países donde se ha llevado a cabo esta misma encuesta.

\section{Conclusiones}

La crisis global del periodismo genera más preguntas que respuestas, y ningún proyecto ha encontrado todavía el modelo ideal para que el periodismo de calidad sea económicamente sostenible. Con el objetivo de clarificar algunas claves de este escenario cambiante y frágil, en este artículo hemos explorado algunas de las claves del slow journalism en Colombia. Para ello, hemos puesto en común datos sobre las audiencias (receptores) y el análisis en profundidad de dos medios colombianos que se encuadran en esta corriente periodística, Arcadia y La Silla Vacía.

Las conclusiones que obtenemos del análisis de datos son varias, pero destaca el amplio conocimiento del concepto de periodismo reposado en Colombia, en comparación con otros países estudiados (España, Argentina, México). Su arraigada e histórica tradición con el periodismo literario podría explicar este fenómeno, aunque sería necesario elaborar un estudio más amplio para conocer los motivos precisos. En este estudio nos ha interesado que los colombianos conocen el concepto y, además, se acercan a este tipo de periodismo con el objetivo de construir una opinión crítica sobre la sociedad. Esta línea es coherente con la filosofía de los dos medios estudiados. Tanto Arcadia como La Silla Vacía proponen un tipo de periodismo crítico y social, aunque cada uno aborde una óptica diferente (cul- 
tura y política). Tampoco coinciden estos dos medios en el soporte al que dan más peso. Conscientes del espacio que el mundo digital está ocupando en nuestras vidas (algo que corroboran los datos de las encuestas), ambos medios han apostado por una edición digital fuerte, pero Arcadia se ha mantenido asimismo fiel a su impecable edición impresa. El análisis comparado de estos dos casos demuestra que el slow journalism tiene un espacio en el mundo digital, aunque tampoco va a desaparecer a corto plazo del papel, un soporte que tradicionalmente ha invitado al análisis reposado.

Las encuestas también corroboran que las audiencias se decantan por la crónica y el análisis cuando piensan en periodismo reposado. La estrategia de Arcadia y La Silla Vacía también sigue esta línea, si bien es cierto que apuestan de manera firme por la innovación y la hibridación de géneros. En la necesidad de innovación hallamos, precisamente, la clave de la puesta en común de datos de los emisores y los receptores obtenidos en este estudio. Los lectores valoran el periodismo reposado, lo conocen y lo consumen. El problema radica en las dificultades para financiarlo, tal y como demuestra el análisis comparativo de los estudios de caso. Por este motivo, los medios enmarcados en el SJ no cesan de innovar en contenidos y métodos de financiación. Su principal reto es diferenciarse de otros medios en calidad y obtener nuevas estrategias de recaudación para subsistir. Los lectores, según constata este estudio, valoran este esfuerzo. Todo apunta a que el periodismo de calidad tiene en Colombia un espacio en el futuro digital e impreso. Sin embargo, parece que la dimensión, sostenibilidad y fortaleza de ese espacio tardará todavía en esclarecerse.

\section{Referencias}

Albalad, J. M. y Rodríguez, J. M. (2015). Retro Report, un modelo de periodismo lento audiovisual. En Sabes, F. y Verón, J. J. (coords.). Innovación y cambio en la comunicación postindustrial. Zaragoza: Asociación de Periodistas de Aragón.

Amado, A. (ed.) (2007). Periodismo de calidad: Debates y desafíos. Buenos Aires: La Crujía y Fopea. 
Anderson, C. W., Bell E. y Shirky C. (2013). Post-industrial journalism: Adapting to the present. Nueva York: Tow Center for Digital Journalism, Columbia University.

Angulo, M. (coord.) (2014). Crónica y mirada. Aproximaciones al periodismo narrativo. Madrid. Libros del K.O.

Ball, B. (2016). Multimedia, slow journalism as process, and the possibility of proper time. Digital Journalism, 4(4), 432-444. DOI: https:// doi.org/10.1080/21670811.2015.1114895

Barranquero Carretero, A. y Jaurrieta Bariain, G. (2016). Slow journalism in Spain: New magazine startups and the paradigmatic case of Jot Down. Journalism Practice, 10(4), 521-538. DOI: https://doi.org /10.1080/17512786.2015.1124729

Barranquero Carretero, A. (2013). Slow media. Comunicación, cambio social y sostenibilidad en la era del torrente mediático. Palabra Clave, 16(2), 419-448. DOI: https://doi.org/10.5294/pacla.2013.16.2.6

Benaissa, S. (2017). El Slow Journalism en la era de la “infoxicación”. Doxa. Comunicación, 25, 129-148. DOI: https://doi.org/10.31921/doxacom.n25a6

Bernal, S. y Chillón, L. A. (1985). Periodismo informativo de creación. Barcelona: Mitre.

Bishop, C. (2015). Slow Journalism: Deep storytelling in the digital age. National Geographic. Recuperado de: https://blog.nationalgeographic.org/2015/01/07/slow-journalism-deep-storytelling-inthe-digital-age/

Bonano, M. (2014). Tendencias del periodismo narrativo actual. Las nuevas formas de contar historias en revistas y cronistas latinoamericanos de hoy. Questión, 1(43), 40-50. 
Callegaro, A. y Lago, M. C. (2012). La crónica latinoamericana: cruce entre literatura, periodismo y análisis social. Quórum Académico, $9(2), 246-262$.

Cuartero Naranjo, A. (2019). Un periodismo a otra velocidad: el libro como formato periodístico en la nueva generación de periodistas narrativos españoles. Estudios sobre el Mensaje Periodístico, 25(2), 747766. DOI: https://doi.org/10.5209/esmp.64800

De Pablos, J. M. y Mateos, C. (2004). Estrategias informativas para acceder a un periodismo de calidad, en prensa y TV. Ámbitos, 11 12, 341-365. Recuperado de: https://www.redalyc.org/articulo. oa? $\mathrm{id}=16801219$

Dowling, D. (2016). The business of slow journalism. Deep storytelling's alternative economies. Digital Journalism, 4(4), 530-546. DOI: https://doi.org/10.1080/21670811.2015.1111769

Drok, N. y Hermans, L. (2016). Is there a future for slow journalism? The perspective of younger users. Journalism Practice, 10(4), 539-554. DOI: https://doi.org/10.1080/17512786.2015.1102604

Goikoetxea Bilbao, U.y Ramírez de la Piscina, T. (2019). Revistas Jot Down, Anfibia y Panenka: tres formas audaces de entender el periodismo narrativo digital en plena crisis del papel. Revista Latina de Comunicación Social, 74, 692-715. DOI: https://doi.org/10.4185/ RLCS-2019-1352

Gómez Mompart, J. L. (2009). From quality journalism to speculative journalism. Transfer, Journal of Contemporary Culture, 6, 55-61.

Gómez Mompart, J. L. et al. (2015). Spanish Journalists and the Loss of News Quality: Professional Judgements. (Los periodistas españoles y la pérdida de la calidad de la información: el juicio profesional.) Comunicar, 45, 143-150. DOI: https://doi.org/10.3916/ C45-2015-15 
Gorosarri, M. G. (2011). Albisteen Kalitatea (Research on Basque Media's News Quality). Leioa: UPV/EHU.

Greenberg, S. (2012). Slow journalism in the digital fast lane. En Lance, K. R. y Tulloch, J., Global Literary Journalism: Exploring the Journalistic Imagination (pp. 381-393). Nueva York: Peter Lang.

Gripsrud, J.y Weibull, L. (2010). Media, markets \& public spheres: European media at the crossroads. Bristol: Intellect.

Herrscher, R. (2009). Periodismo narrativo. Manual para contar la realidad con las armas de la literatura. Santiago de Chile: RIL, Universidad Finis Terrae.

Hoyos, J.J. (2009). La pasión de contar: el periodismo narrativo en Colombia, 1638-2000. Medellín: Hombre Nuevo, Universidad de Antioquia.

Jaramillo Agudelo, D. (ed.) (2012). Antología de crónica latinoamericana actual. Madrid: Alfaguara.

Lago, M. C. y Callegaro, A. (2012): Crónica latinoamericana. Cruce entre literatura, periodismo y análisis social. Madrid: Editorial Académica Española. DOI: https://doi.org/10.5216/CEI.v15i1.22496

Le Masurier, M. (2015). What is slow journalism? Journalism Practice, 9(2), 138-152.DOI: https://doi.org/10.1080/17512786.2014.916471

Le Masurier, M. (2016). Slow journalism. Digital Journalism 4(4), 405-413. DOI: https://doi.org/10.1080/21670811.2016.1139904

Lindlof, T. R. y Taylor B. C. (2017). Sensemaking: Qualitative data analysis and interpretation. Qualitative communication research methods. 4 ed. Nueva York: SAGE.

Llop, P. (2014). El auge del longform journalism. Cuéntamelo despacio, que tengo prisa. [Documento en línea]. Recuperado de: http:// www.2ip.es/wp-content/uploads/2014/07/SlowJournalism.pdf 
López Pan, F. (2010) Periodismo literario: entre la literatura constitutiva y la condicional. Ámbitos 19, 97-116. DOI: https://doi. org/10.12795/Ambitos.2010.119.06

Mendelson, A. L. y Creech, B. (2016). "Make Every Frame Count." The practice of slow photojournalism and the work of David Burnett. Digital Journalism, 4(2), 512-529. DOI: https://doi.org/10.1080 /21670811.2015.1124727

Neveu, E. (2016). On not going too fast with slow journalism. Journalism Practice, 10(4), 448-460. DOI: https://doi.org/10.1080/17512 786.2015.1114897

Palacios, M. y Díaz Noci, J. (2009). Ciberperiodismo: métodos de investiogación. Una aproximación multidisciplinar en perspectiva comparada. Bilbao: Universidad del País Vasco.

Palau-Sampio, D. (2018). Reframing Central American migration from narrative journalism. Journal of Communication Inquiry, 43(2). DOI: https://doi.org/10.1177/0196859918806676

Pellegrini, S. y Múgica, M. C. (2006). Valor agregado periodístico (VAP): La calidad periodística como un factor productivo en un entorno medial complejo. Palabra Clave, 9(1), 11-28.

Puerta, A. (2011). El periodismo narrativo o una manera de dejar huella de una sociedad en una época. Anagramas, 9(18), 47-60. DOI: https://doi.org/10.22395/angr.v9n18a3

Ramírez de la Piscina, Tx., Gz. Gorosarri, M., Aiestaran, A., Zabalondo, B. y Agirre, A. (2014). Periodismo de calidad en tiempos de crisis: un análisis de la evolución de la prensa europea de referencia (2001-2012). Revista Latina de Comunicación Social, 69, 248-274.

Rauch, J. (2011). The origin of slow media: Early diffusion of a cultural innovation through popular and press discourse, 2002-2010. 
Transformations, 20. Recuperado de: http://www.transformationsjournal.org/issue-20/

Rodríguez Rodríguez,J. M. y Albalad Aiguabella J. M. (2012). Nuevas ventanas del periodismo narrativo en español: del big bang del boom a los modelos editoriales emergentes. Textual \& Visual Media, 5, 287-310.

Rosenberg, H. y Feldman, C. S. (2008). No time to think. The menace of media speed and the 24-hour news cycle. Nueva York: Continuum.

Rosique-Cedillo, G. y Barranquero-Carretero, A. (2015). Periodismo lento (slow journalism) en la era de la inmediatez. Experiencias en Iberoamérica. El Profesional de la Información, 24(4), 451-462. DOI: https://doi.org/10.3145/epi.2015.jul.12

Sierra Caballero, F. (2012). Do boom ao Big Bang: a ruptura do cânone e a recepção do novo jornalismo latinoamericano na Espanha. Revista Comunicação Midiática, 7(3), 14-30.

Sierra Caballero, F. y López Hidalgo, A. (2016). Periodismo narrativo y estética de la recepción. La ruptura del canon y la nueva crónica. Estudios sobre el Mensaje Periodístico, 22(2), 915-935. DOI: https:// doi.org/10.5209/ESMP.54243

Waisbord, S. (2018). Truth is what happens to news. Journalism Studies, 19(13), 1866-1878. DOI: https://doi.org/10.1080/146167 0X.2018.1492881

Wimmer, R.D. y Dominick J. R. (1996). La investigación cientifica de los medios de comunicación: una introducción a sus métodos. Barcelona: Bosch.

Wolfe, T. (1973). The new journalism. Nueva York: Harper \& Row.

Yin, R. K. (1992). The case study method as a tool for doing evaluation. Current Sociology, 40(1), 121-137. 